\title{
Systemic cytokines are elevated in a subset of patients with irritable bowel syndrome but largely unrelated to symptom characteristics
}

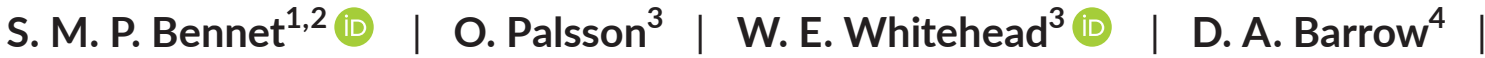 \\ H. Törnblom ${ }^{1}$ | L. Öhman ${ }^{1,2} \mid$ M.Simrén ${ }^{1,3} \mid$ M. A. L. van Tilburg ${ }^{3,5,6}$
}

${ }^{1}$ Institute of Medicine, University of Gothenburg, Gothenburg, Sweden

${ }^{2}$ Institute of Biomedicine, University of Gothenburg, Gothenburg, Sweden

${ }^{3}$ Center for Functional Gastrointestinal and Motility Disorders, Division

of Gastroenterology and

Hepatology, University of North Carolina, Chapel Hill, NC, USA

${ }^{4}$ Center for Oral and Systemic Diseases, School of Dentistry, University of North Carolina, Chapel Hill, NC, USA

${ }^{5}$ School of Social Work, University of Washington, Seattle, WA, USA

${ }^{6}$ College of Pharmacy \& Health Sciences, Campbell University, Buies Creek, NC, USA

\section{Correspondence}

Sean Bennet, Department of Internal

Medicine and Clinical Nutrition, University

of Gothenburg, Gothenburg, Sweden.

Email: sean.bennet@gu.se

Funding information

This study was supported by Takeda, an NIH funding NIDDK grant (RO1 DK31369), the Swedish Medical Research Council (grants 13409, 21691 and 21692), AFA Insurance, an unrestricted research grant from Ferring Pharmaceuticals, and by the Faculty of Medicine, University of Gothenburg.

\begin{abstract}
Background: Serum levels of pro-inflammatory cytokines tend to be increased in irritable bowel syndrome (IBS) patients, or subgroups thereof. Still, the link between cytokine levels and IBS symptoms is unclear. We aim to determine systemic cytokine levels in IBS patients and healthy subjects (HS), confirm the presence of a subset of patients with an increased immune activity and to establish if cytokines are linked to IBS symptoms and pathophysiological factors.

Methods: Serum levels of interleukin (IL)-1 $\beta$, IL-6, IL-8, tumor necrosis factor (TNF), and IL-10 were measured. All subjects reported IBS symptoms using validated questionnaires and underwent colonic sensorimotor testing. Multivariate supervised orthogonal partial least squares-discriminant analysis (OPLS-DA) and unsupervised principal component analysis (PCA) and hierarchical cluster analysis (HCA) were implemented.

Key Results: Irritable bowel syndrome patients $(n=246)$ had higher serum levels of IL-1 $\beta$, IL-6, IL-8, TNF, and IL-10 compared to HS ( $n=21)$; however, serum cytokine profiles could not discriminate patients from HS. Moreover, cytokine levels were not correlated with symptoms among patients. Supervised OPLS-DA identified 104 patients (40\% of patients) and unsupervised HCA analysis identified 49 patients (20\%) with an increased immune activity indicated by elevated levels of serum cytokines compared to HS and the other patients. However, irrespective of how patients with increased immune activity were identified they were symptomatically similar to patients with no indication of increased immune activity.

Conclusions \& Inferences: Serum cytokines are elevated in IBS patients compared to HS. Immune activation characterizes a subset of patients, but modest associations between cytokine profile and symptoms suggest immune activity does not directly influence symptoms in IBS.
\end{abstract}

KEYWORDS

cytokines, IBS, symptom 


\section{1 | INTRODUCTION}

Irritable bowel syndrome (IBS) is characterized by altered bowel habits and abdominal pain. ${ }^{1,2}$ Approximately $5 \%-15 \%$ of adults in the western world are afflicted with this combination of gastrointestinal (GI) symptoms and are diagnosed with IBS. ${ }^{3-5}$ Irritable bowel syndrome patients have a recognized heterogeneity and differ in aspects such as severity and constellation of symptoms, abruptness of onset and possible triggers, ${ }^{6}$ making effective subgrouping a challenging but necessary task. ${ }^{7}$

The well-established clinical practice of subtyping according to predominant bowel habit, ie, constipation (IBS-C), diarrhea (IBS-D), or mixed (IBS-M), is both tangible and non-invasive. However, the apparent fluctuation in stool consistency of an individual makes subtyping difficult. ${ }^{8}$ Moreover, the high heterogeneity means that although 2 patients are subtyped as IBS-D, they may have different underlying diarrhea mechanisms, such as increased colonic bile acid exposure $^{9}$ or the osmotic effect of certain foods which draw fluid into the bowel. ${ }^{10}$ Instead, subgrouping based on symptom pathogenesis or pathophysiology may be preferable and result in better, more targeted treatment. ${ }^{11}$

Although the pathophysiology of IBS is complex, incompletely understood, and the mechanisms behind symptom generation are potentially different between patients, it has been suggested that a subset of patients have an increased systemic immune activity. ${ }^{12-16}$ One means to assess systemic immune activity is to measure the levels of serum glycoproteins (cytokines) that regulate the functions of the immune system. A range of cytokines, for example interleukin 12 (IL-12), interferon-gamma (IFN- $\gamma$ ), and transforming growth factor beta (TGF- $\beta$ ) have been investigated in relation to IBS in previous studies yet conflicting results are prevalent. ${ }^{16-18}$ However, pro-inflammatory IL-1 $\beta$, IL-6, IL-8, tumor necrosis factor-alpha (TNF), and anti-inflammatory IL-10, important cytokines in the immune system ${ }^{19-21}$ are commonly reported as being significantly altered in IBS patients compared to healthy subjects (HS). ${ }^{22,23}$ Moreover, associations between serum levels of cytokines, eg, IL-6 and symptoms such as abdominal pain and discomfort have been demonstrated, ${ }^{24,25}$ but many studies require further validation and/or are performed on small IBS cohorts which may not encompass the full spectrum of patients. ${ }^{7}$ In a recent study by our group, IBS patients and HS could not be discriminated from each other through multivariate statistical analysis based upon their cytokine profiles; however, we detected an "immuno-active" subset of patients particularly defined by elevated serum cytokine levels of IL- 6 and IL-8. ${ }^{16}$ In that study, only modest associations between levels of pro-inflammatory cytokines and symptoms, and pathophysiology were detected.

Therefore, we hypothesize that within each IBS cohort a subset of patients with increased immune activity, possibly associated with symptoms, is present when compared to other IBS patients and HS. Thus, the aim of this study was to determine the serum cytokine profiles from another cohort of well-characterized IBS patients and HS, and to confirm the existence of the previously proposed "immunoactive" subset of patients. Additionally, we aimed to investigate the

\section{Key Points}

- Increased systemic immune activity has been demonstrated in irritable bowel syndrome (IBS) patients compared to healthy subjects yet may only be relevant in a subset of patients.

- Two independent statistical methods identified a patient subset with increased serum cytokine levels; however, neither systemic levels of cytokines, nor profiles thereof, are associated with the severity of IBS symptoms.

- The cause for the increased immune activity in these patients requires elucidation and may still be relevant in the pathogenesis of their IBS.

relationship between serum cytokines and clinical characteristics of IBS patients.

\section{MATERIALS AND METHODS}

\section{1 | Study cohort and material collection}

Irritable bowel syndrome patients who fulfilled the Rome II criteria for IBS had abdominal pain or discomfort for a minimum of 1 day a week occurring in the last 3 months. Each patient received a physician diagnosis and was included over the last decade at the Center for Functional Gastrointestinal and Motility Disorders, University of North Carolina at Chapel Hill in the USA. All patients were recruited by physician referrals or advertisements and were screened by telephone. Exclusion criteria included presence of inflammatory bowel disease, lactose malabsorption, celiac disease, heart disease, or diabetes mellitus, history of GI surgery (other than appendectomy or cholecystectomy), having been on antibiotics for the prior 2 weeks, or being pregnant at the time of study. Patients were subtyped according to the Rome II and Rome III criteria. ${ }^{26,27}$ Healthy subjects were recruited and demographic variables including age and race were adjusted to approximate the IBS sample. Healthy subjects had to have no abdominal pain except menstrual, no GI disorders by self-report, and use no GI medications. They should take laxative or antidiarrheals no more than once per month. They were recruited by advertisement and were paid for their participation. All study subjects were advised to avoid ibuprofen, aspirin, or other pain medication during the 24 hours before serum collection. All subjects provided written informed consent and the study was approved by the Institutional Review Board of the University of North Carolina.

\section{2 | Serum cytokine analysis}

Blood was collected in serum-separating tubes from subjects during the period between waking up and before they had eaten breakfast. Within 2 hours of venipuncture, the samples were left to stand for 30 minutes before being spun at $1500 \mathrm{~g}$ for 10 minutes at 
room temperature. The serum samples were then frozen and maintained at $-80^{\circ} \mathrm{C}$ (except for transport to the lab on dry ice) until the morning of analysis when they were allowed to thaw at room temperature and then assayed according to the reagent manufacturer's assay protocol. Based on prior literature describing alterations between IBS patients and HS, the levels of pro-inflammatory IL-1 $\beta$, IL-6, IL-8, and TNF and anti-inflammatory IL-10 were focused on $^{22,23}$ and assessed through high sensitivity multiplex assays (BioPlex 200; Bio-Rad, Hercules CA, USA, using FMAP reagents from R\&D Systems, Minneapolis, MN, USA). Lowest detection limits given as $\mathrm{pg} / \mathrm{mL}$ were set for each target according to manufactures guidelines and were as follows 0.18 for IL-1 $\beta, 0.31$ for IL-6, 0.07 for IL-8, 0.54 for TNF, and 0.24 for IL-10. Measurements which were under the detection limit threshold were set as the detection limit threshold.

\section{3 | Clinical and pathophysiological assessments}

\subsection{1 | Colonic sensory and motility testing}

Sensory and motility testing was performed using a combined manometry and a barostat catheter (Model C7- CB- 00256; Mui Scientific, Mississauga, ON, Canada), positioned in the descending colon $(n=67)$ or rectum ( $n=197$ ). Following catheter placement, the subject rested for 90 minutes before testing began. No sedation was used during the catheter placement and the subjects came to the laboratory fasting. For the purpose of this study, the pain and urge threshold obtained using an ascending method of limits protocol were used as a measure of colorectal sensitivity (Dual Drive Barostat, Distender Series II; G\&J Electronics Inc, Toronto, ON, Canada), and to be able to combine colonic and rectal thresholds, Z-scores (defining how many standard deviations a value is from the mean) were calculated. For motility assessment, phasic contractions in the fasting state, during balloon distention (10 minutes at $20 \mathrm{~mm} \mathrm{Hg}$ ), during the recovery period after intraluminal distention, and 30 minutes following an $810 \mathrm{kcal}$ meal, were analyzed and motility indices for these 4 periods were calculated (Sandhill Scientific, Highlands Ranch, CO, USA). For further details, please see the study by Kanazawa et al. ${ }^{28}$

\section{4 | Individual assessment questionnaires}

All study subjects in this cohort completed the following selfassessment questionnaires to assess coping and the severity of psychological, GI, and extraintestinal symptoms.

\subsection{1 | IBS severity scoring system}

Used in the assessment of the perceived severity of IBS symptoms, this questionnaire comprises 5 questions using visual analog scales. ${ }^{29}$ The interference of IBS symptoms with daily life, severity of abdominal distention, severity of abdominal pain, frequency of abdominal pain, and dissatisfaction with bowel habits are included. A range from 0 (no symptoms) to 500 (maximum severity) can be achieved with patients falling into subgroups of mild (75175), moderate (175-300), or severe (>300) IBS symptoms.

\subsection{2 | Irritable bowel syndrome-quality of life measure}

Thirty-four IBS-specific quality of life questions use a 5-point Likert scale to assess how much during the past month (30 days), the statement described the feelings of the respondent: not at all, slightly, moderately, quite a bit, and extremely or a great deal. All items were sum-scored to and transformed to a $0 \pm 100$ scale ranging from 0 (poor quality of life) to 100 (maximum quality of life) as previously described. ${ }^{30,31}$

\subsection{3 | Recent physical symptoms questionnaire}

Twenty-six non-Gl physical symptoms that are significantly more common in IBS patients compared to healthy individuals are assessed in the RSPQ. ${ }^{32}$ The subject is asked to indicate "how frequently you have experienced each symptom in the past month" using the response options $0=$ "never or only once," $1=$ "less than 1 day a week," 2 = "at least 1-2 days a week," 3 = "most days," and 4 = "every day." The RSPQ score is thus a measure of the psychological tendency to report multiple physical symptoms experienced with higher frequency than "never or only once" in the past month. The recent physical symptoms questionnaire (RPSQ) somatization score is calculated as the total number of different symptoms on the questionnaire that the respondent reports with a higher frequency than "never or only once."

\subsubsection{Comorbid medical conditions questionnaire}

The comorbid medical conditions questionnaire $(\mathrm{CMCQ})^{32}$ is a selfreported assessment of medical diagnoses received by the study subject. Briefly, the questionnaire requires the study subject to indicate whether they have "ever been diagnosed by a physician" for IBS or any of the 16 non-GI diagnoses. Possible answers are "yes," "no" or "don't know" with the response "don't know" being equated to the "no" response. The total number of "yes" responses gives an index of the subject's number of medical comorbidities and is denoted as the CMCQ score.

\subsection{5 | Brief symptom inventory}

Subjects completed the brief symptom inventory-18 (BSI-18) validated questionnaire in order to measure the degree of psychological distress during the past week. Consisting of a 5-point scale ranging from "not at all" to "extremely" subjects report how much they were bothered by each of the 18 symptoms. Scores of each subscale of anxiety, and depression were used in this study, and converted into standardized scores allowing for BSI-18 scores for women and men to be pooled together for analysis. ${ }^{33}$ 


\subsection{6 | Catastrophizing}

For this study, 6 items from the Coping Strategies Scale ${ }^{34}$ were used to gauge how subjects expressed feelings of hopelessness and the expectation that pain (if they are patients) will become worse. All items were answered on a 6-point scale, ranging from ${ }^{1}$ it is terrible and I feel it is never going to get any better to ${ }^{6}$ I feel like I can't go on.

\section{5 | Data and statistical analysis}

\subsection{1 | Multivariate analysis}

Multivariate factor analyses were applied to serum cytokine level profiles ( $\mathrm{X}$ variables) of IBS patients and HS ( $\mathrm{Y}$ variables) using SIMCA software (Version 13.0.3.0, copyright (C) Umetrics AB). By using multivariate analysis, all $X$ and all $Y$ variables can be simultaneously assessed allowing for identification of any patterns in the dataset. The heterogeneity in IBS is also taken into consideration as those with similar profiles could be easily identified in such multivariate models as compared to univariate analysis. The Hotelling's $T^{2}$ is a multivariate form of Student's $t$ test implemented to define the normal area ellipse corresponding in this study to a $95 \%$ confidence. Respective to the other subjects, those falling outside of this ellipse are broadly defined as explainable outliers having a different profile of measured $X$ variables (serum cytokines). In this cohort, a 95\% confidence ellipse was used meaning any subjects outside of the ellipse were considered potential outliers which deviate from normality and can skew the model. However, not all of these outliers are likely to be "real" outliers. The distance to the model (DmodX) plot indicates how well a subject fits the model with a high DmodX indicating a poor fit and denoting the subject as a weak outlier. If a subject did not fit the model and laid outside of the confidence ellipse, then it was considered a true outlier and was excluded.

Orthogonal partial least squares-discriminant analysis (OPLS-DA) was implemented to investigate if serum cytokine levels ( $X$ variables) could be used to discriminate IBS patients from HS (Y variables). The $R^{2}$ parameter represents the goodness of the fit of the OPLS-DA, while the $Q^{2}$ represents the internal crossvalidation of the model. Although the best possible fit is $R^{2}=1$ and an optimal $Q^{2}$ is 0.7 or higher, when regarding biological variables an $R^{2} \geq 0.5$ and $Q^{2} \geq 0.4$ is considered satisfactory. ${ }^{35} \mathrm{~A}$ loading plot was generated to identify which of the $X$ variables (serum cytokines) had the most power regarding their ability to discriminate HS from IBS patients. Cytokines localizing further away from the center of the $x$-axis contribute more to the discrimination of the 2 groups. A loading scatter plot was generated which may be superimposed over the OPLS-DA to better visualize which cytokines are associated with the groups of the cohort, eg, IBS patients or HS.

Principal component analysis (PCA) including all study subjects was performed in order to identify any discernible groups, trends, or correlations between the subjects. Unsupervised "bottom-up" hierarchical clustering analysis (HCA) was performed to identify clusters of study subjects with similar serum cytokine profiles. Similar to the OPLS-DA, a loading scatter plot of the PCA was generated which may be superimposed over the PCA to better visualize which cytokines are associated with the clusters of the cohort as defined though the HCA.

\subsection{2 | Univariate analysis}

All univariate analyses were performed using the GraphPad Prism Version 6.04 (GraphPad Software Inc., San Diego, CA, USA). When comparing significance in continuous data between 2 groups the Mann-Whitney $U$ test was applied, while the Kruskal-Wallis, followed by Dunn's test was implemented when testing significance between 3 or more groups. Non-parametric Spearman's rank coefficient was used for correlations. The classical 1-stage method was used when comparing multiple parameters to account for the false discovery rate (FDR); corrected $P$-values were denoted as $q$-values. In this study, significance was denoted as a $P$-value of <.05; if the finding was insignificant after correction for multiple comparisons, they were thus described as tendencies. Results in text, tables, and figures are presented as median followed by range shown as 25 th and 75 th percentile.

\section{3 | RESULTS}

\subsection{Clinical characteristics of study subjects}

A total of 267 study subjects were included comprising 246 IBS patients and $21 \mathrm{HS}$. The median age of the patients included was 33.0 years (25.0-45.0 years) and of the HS 30.0 years (26.543.5 years). Of the questionnaire parameters assessed, IBS severity scoring system (IBS-SSS), IBS quality of life measure (IBS-QOL), RSPQ, CMCQ, and BSI anxiety and depression differed between IBS patents and HS (Table 1). Of the IBS patients, $14 \%$ were defined as IBS-C, $21 \%$ as IBS-D, and $65 \%$ as IBS-M, and among these patients, $11 \%$ reported onset of their IBS symptoms after an infection (PI-IBS) (Table 1). Of the 267 study subjects, 15 were identified as explainable outliers when using a $95 \%$ Hotelling's $T^{2}$ confidence ellipse. After consulting the DModX, 2 of the 15 subjects were IBS patients which were not modeled well and so were considered true outliers and removed to reduce risk of skewing the model. Additionally, $1 \mathrm{HS}$ was also identified as a true outlier due to abnormally high levels of anxiety and depression and levels of IL-6; hence 264 subjects were included in the subsequent analyses.

\subsection{Levels of serum cytokines differs between IBS patients and HS}

Serum levels of IL-6, IL-8, and TNF were detectable in all patients and HS, while levels of IL-10 were below the detection limit in 11 patients (4.5\%) and $11 \mathrm{HS}(55 \%)$ and levels of IL-1 $\beta$ were below the detection limit in 14 patients (5.7\%) and 13 HS (65\%). Comparing the 
TAB LE 1 Demographic and clinical characteristics of IBS patients and healthy subjects

\begin{tabular}{|c|c|c|c|c|c|c|}
\hline & IBS & IBS-C & IBS-D & IBS-M & Healthy & $P$-value ${ }^{a}$ \\
\hline Total (n) & 246 & 35 & 51 & 160 & 21 & \\
\hline Female (\%) & 77.2 & 82.9 & 64.7 & 80 & 100 & .02 \\
\hline Age, years & $33.0(25.0-45.0)$ & $36(24-43)$ & $37(27-45)$ & $31(24-46)$ & $30.0(26.5-43.5)$ & .6 \\
\hline Caucasian (\%) & 70.5 & 40.0 & 84.3 & 72.8 & 57.1 & \\
\hline PI-IBS (\%) & 10.9 & 17 & 5.9 & 11.3 & $\mathrm{n} / \mathrm{a}$ & \\
\hline IBS-SSS & 262 (187-325) & $295(210-340)$ & $240(187-310)$ & $280(205-330)$ & $0(0-0)$ & $<.0001$ \\
\hline IBS-QOL & 75 (62-84.6) & $73.5(60.3-89)$ & $77.9(60.3-83)$ & $74.3(63.2-82.5)$ & $100(100-100)$ & $<.0001$ \\
\hline RSPQ & $11(7-15)$ & $11(7-14)$ & $9(6-13)$ & $12(8-15)$ & $1(0-6)$ & $<.0001$ \\
\hline CMCQ & $2(1-3)$ & $2(1-3)$ & $1(0-3)$ & $2(1-4)$ & $0(0-1)$ & .001 \\
\hline BSI anxiety & $52(45-60)$ & $48(38-59)$ & $48(45-60)$ & $52(45-61)$ & $38(38-38)$ & $<.0001$ \\
\hline BSI depression & $49(42-61)$ & $48(40-58)$ & $48(42-61)$ & $56(42-61)$ & $40(40-40)$ & $<.0001$ \\
\hline
\end{tabular}

Data shown as median (25\%-75\%).

IBS-C: IBS with constipation; IBS-D: IBS with diarrhea; IBS-M: IBS with mixed constipation and diarrhea; PI-IBS: post infectious IBS; IBS-SSS: irritable bowel syndrome severity scoring system; IBS-QOL: quality of life; RPSQ: recent physical symptoms questionnaire; BSI: brief symptom inventory; CMCQ: comorbid medical conditions questionnaire; $\mathrm{n} / \mathrm{a}$ : not applicable.

${ }^{a}$ Mann-Whitney $U$ test between IBS and healthy.

systemic levels of cytokines between included patients and HS revealed that pro-inflammatory IL-1 $\beta$, IL-6, TNF, and anti-inflammatory IL-10 were higher in patients, while IL-8 only tended to be higher in patients compared HS (Table 2). Although higher levels of IL-1 $\beta$, IL-6, and IL-10 were found in IBS-C, IBS-D, and IBS-M compared to HS, there were no differences between the patient subtypes (Table 3). Serum levels of TNF were only higher in IBS-D compared to HS, while levels of IL-8 were comparable between the IBS subtypes and
HS (Table 3). No differences in measured cytokines were detected between self-reported PI-IBS and non-PI-IBS (Table S1). Finally, IL-8 levels were higher in patients with 2 or more comorbid medical conditions, which was not seen for any of the other measured cytokines (Table S2).

In the total cohort, levels of IL-6 showed positive correlations with both RPSQ somatization $(r=.15, P=.01)$ and IBS-SSS $(r=.14$, $P=.02)$; however, these disappeared after correction for multiple

TAB LE 2 Serum cytokine levels in IBS patients and healthy subjects

\begin{tabular}{llll} 
Cytokine $(\mathrm{pg} / \mathrm{mL})$ & IBS $(\mathrm{n}=244)$ & Healthy $(\mathrm{n}=20)$ & $P_{\text {-value }}{ }^{\mathrm{a}}$ \\
\hline IL-1 $\beta$ & $0.69(0.45-1.05)$ & $0.18(0.18-0.62)$ & $<.00001$ \\
\hline IL-6 & $2.31(1.58-3.57)$ & $0.93(0.65-2.17)$ & $<.00001$ \\
\hline IL-8 & $9.33(6.56-12.3)$ & $8.08(3.9-9.57)$ & .07 \\
IL-10 & $1.02(0.65-1.59)$ & $0.24(0.24-0.58)$ & $<.00001$ \\
\hline TNF & $9.57(7.8-12.2)$ & $7.79(5.33-9.85)$ & .01 \\
\hline
\end{tabular}

Data shown as median (25\%-75\%).

IBS: irritable bowel syndrome; IL: interleukin; TNF: tumor necrosis factor.

a Mann-Whitney $U$ test.

TAB LE 3 Serum cytokine levels in IBS bowel habit subtypes and healthy subjects

\begin{tabular}{llllll} 
Cytokine $(\mathrm{pg} / \mathrm{mL})$ & IBS-C $(\mathbf{n}=35)$ & IBS-D $(\mathbf{n}=51)$ & IBS-M $(\mathbf{n}=160)$ & Healthy $(\mathbf{n}=20)$ & $P$-value ${ }^{\mathrm{a}}$ \\
\hline IL-1 $\beta$ & $0.62(0.47-0.82)^{(\mathrm{H})}$ & $0.66(0.44-1.25)^{(\mathrm{H})}$ & $0.71(0.45-1.11)^{(\mathrm{H})}$ & $0.18(0.18-0.62)$ & .0002 \\
\hline IL-6 & $2.43(1.47-3.89)^{(\mathrm{H})}$ & $2.24(1.64-3.8)^{(\mathrm{H})}$ & $2.3(1.57-3.41)^{(\mathrm{H})}$ & $0.92(0.65-2.17)$ & .0001 \\
\hline IL-8 & $10.1(7.79-12.9)$ & $9.27(6.16-11.8)$ & $8.97(6.54-12.2)$ & $8.08(3.9-9.57)$ & .08 \\
IL-10 & $1.01(0.72-1.75)^{(\mathrm{H})}$ & $0.92(0.66-1.58)^{(\mathrm{H})}$ & $1.04(0.65-1.59)^{(\mathrm{H})}$ & $0.24(0.24-0.58)$ & $<.0001$ \\
\hline TNF & $9.37(7.91-11.9)$ & $10.4(7.83-13.2)^{(\mathrm{H})}$ & $9.52(7.83-11.9)$ & $7.79(5.33-9.85)$ & .04 \\
\hline
\end{tabular}

Data shown as median (25\%-75\%).

IBS: irritable bowel syndrome; IBS-C: IBS with constipation; IBS-D: IBS with diarrhea; IBS-M: IBS with mixed constipation and diarrhea; IL: interleukin; TNF: tumor necrosis factor; $(\mathrm{H})$ : Post hoc significant differences, higher than healthy.

${ }^{a}$ Kruskal-Wallis test, followed by Dunn's multiple comparisons test. 
comparisons ( $q=0.18$, respectively). A negative correlation was found between levels of IL-10 and quality of life which held true after FDR ( $r=-.19, P=.002, q=0.04)$. Although lost after FDR, IL-10 correlated positively with IBS-SSS $(r=.15, P=.01, q=0.1)$. Serum levels of the other measured cytokines did not correlate with any of the other clinical and pathophysiological parameters in IBS patients and HS in this study (Table S3).

\section{3 | Serum cytokine profiles do not distinguish patients from HS, but can be used to identify a subset of IBS patients with increased immune activity}

Following the same methodology as in our previous study, a supervised OPLS-DA was performed. ${ }^{16}$ In corroboration with our previous findings, the overlap of patients and HS and low model indices demonstrated that the 2 groups did not differ regarding overall serum cytokine profiles $\left(R^{2}=0.6 Q^{2}=0.2\right.$; Figure $\left.1 \mathrm{~A}\right)$. The loading plot revealed that of the 4 cytokines, IL- 6 and IL-10 had marginally more discriminatory power than IL-8 and TNF, and in line with the univariate analysis showed indication to be higher in IBS patients compared to HS (Figure 1B). Furthermore, a subset of 104 patients ( $40 \%$ of the IBS patients) was characterized as having an increase in immune activity (immuno-active), localized to the left of the HS in the OPLS-DA plot (Figure 1C). By comparison, the patients who colocalized with the HS were most likely to display normal level of immune activity (immuno-normal) and thus have "healthy-like" levels of serum cytokines. Univariate analyzes were performed following the identification of the immuno-active and immuno-normal IBS subsets among the patients during the OPLS-DA (Figure 1C). As expected, the immuno-active patients had higher serum levels of IL-1 $\beta$, IL-6, IL-8, TNF, and IL-10 compared to the immuno-normal subset of patients (Table S4).

\subsection{Four distinct clusters identified through unsupervised multivariate analysis of serum cytokine profiles of IBS patients and HS}

We next performed an unsupervised PCA and HCA on the dataset with the aim of confirming the presence of a subset of IBS patients with an increased immune activity profile as identified in the OPLS-DA above.

The PCA corroborated with the OPLS-DA showing high heterogeneity among the patients, but a more homogeneous cytokine profile among the HS, signified by the majority of these subjects colocalizing in the top left quadrant of the plot (Figure 2A). We thus took the whole cohort of patients and HS and performed unsupervised HCA and determined 4 clusters of subjects; cluster 1 (7 HS and 18 patients), cluster 2 (11 HS and 105 patients), cluster 3 (2 HS and 72 patients), and cluster 4 (0 HS and 49 patients; Figure 2B). The clusters are highlighted in the PCA plot (Figure $2 \mathrm{C}$ ) allowing for the loading plot (Figure 2D) to be used to reveal that subjects of cluster 1 (to the left in the score plot) were characterized by having the lowest level of cytokines. Cluster 2 and 3 occupied a more central part of the score plot, while cluster 4 can be found to the right in the score plot, and characterized by having the highest level of cytokines. Analysis of the 4 clusters revealed that cluster 4, which was comprised of only patients, had the highest serum levels of IL-1 $\beta$, IL-6, IL-8, TNF, and IL-10 compared to the other 3 clusters (Table 4). Looking at which of the subjects in these clusters had previously been defined as immuno-active in the OPLS-DA, it was revealed that the immuno-active group was composed of no patients from cluster 1 , but of patients from clusters 2 ( $n=22 ; 21 \%$ of cluster 2 ), 3 ( $n=36 ; 50 \%$ of cluster 3 ), and 4 ( $n=48 ; 98 \%$ of cluster 4 ). Thus, by using an unsupervised method of cluster analysis, a subset of patients comprising $20 \%$ (cluster 4 , $n=49$ IBS patients) of the patients in total cohort, were identifiable as having increased immune activity compared to the rest of the patients and HS.

\section{5 | Symptom severity and colorectal sensorimotor function in IBS are largely independent of systemic levels of cytokine levels}

Irrespective of the supervised or unsupervised methods used for their identification, we next examined if patients with increased immune activity had altered symptom profile or colorectal sensorimotor function. No significant differences were identified when comparing immuno-active and immuno-normal patients regarding IBS symptom severity, extraintestinal symptoms, psychological symptoms, coping, or colorectal sensorimotor function (Table 5). Few differences were also seen when comparing symptoms and colorectal sensorimotor function between patients within each cluster (cluster 1 [18 IBS], cluster 2 [105 IBS], cluster 3 [72 IBS], and cluster 4 [49 IBS]; Table 6). Cluster 1 was found to have a significantly lower rectal pain threshold compared to cluster $2(P=.02)$, cluster 3 $(P=.01)$, and cluster $4(P=.02)$, and cluster 1 also had a significantly lower Catastrophizing score than cluster $3(P<.01)$, but otherwise no differences between the clusters were detected (Table 5). We thus demonstrated that irrespective of using the supervised subtyping method or the unsupervised clustering method, patients identified as having increased immune activity could not be distinguished from the rest of the patient cohort based on symptoms or colorectal sensorimotor function.

\section{DISCUSSION}

In this study, we have demonstrated that systemic levels of cytokines indicative of immune activation are higher in IBS patients compared to HS. Moreover, while patients could not be differentiated from HS through multivariate analysis of serum cytokine profiles, a cluster of patients with a distinctly increased immune activity was identified. However, as differences in symptom pattern were not seen between the clusters of IBS patients based on immune activity, a direct effect of immune activity on symptoms in IBS is not supported by our findings. Still, increased activity of the immune system may be relevant 
(A)

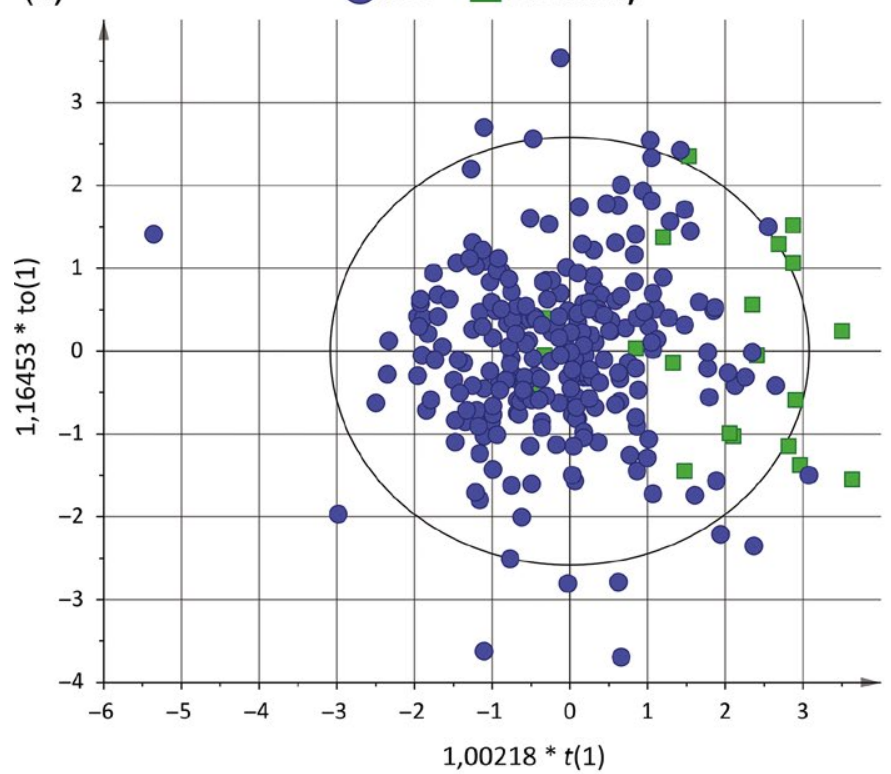

(B)

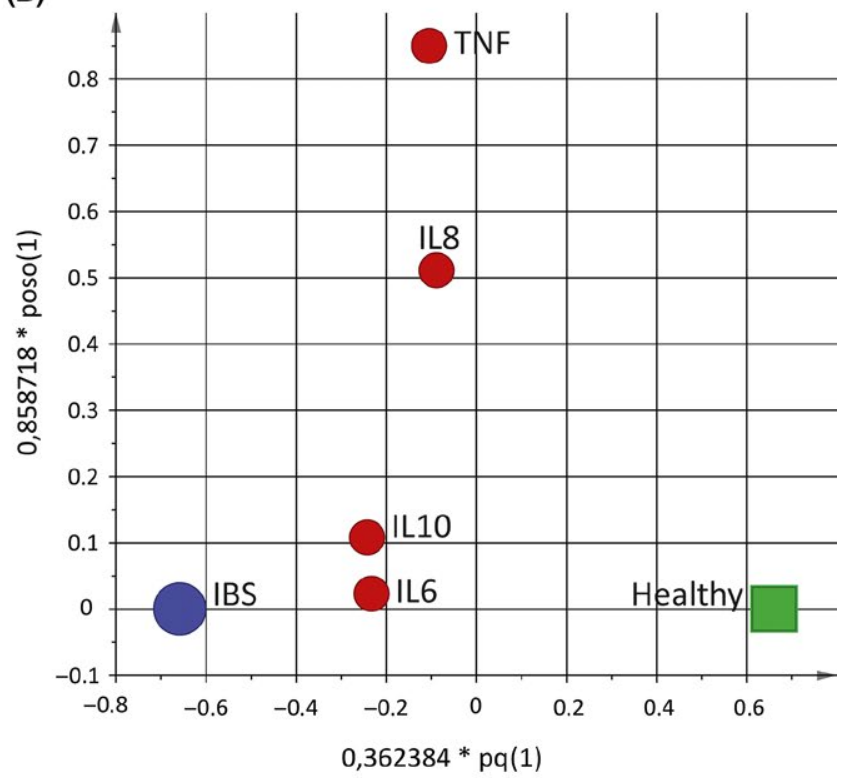

(c) $\bigcirc$ Immuno-active IBS $\bigcirc$ Immuno-normal IBS $\square$ Healthy

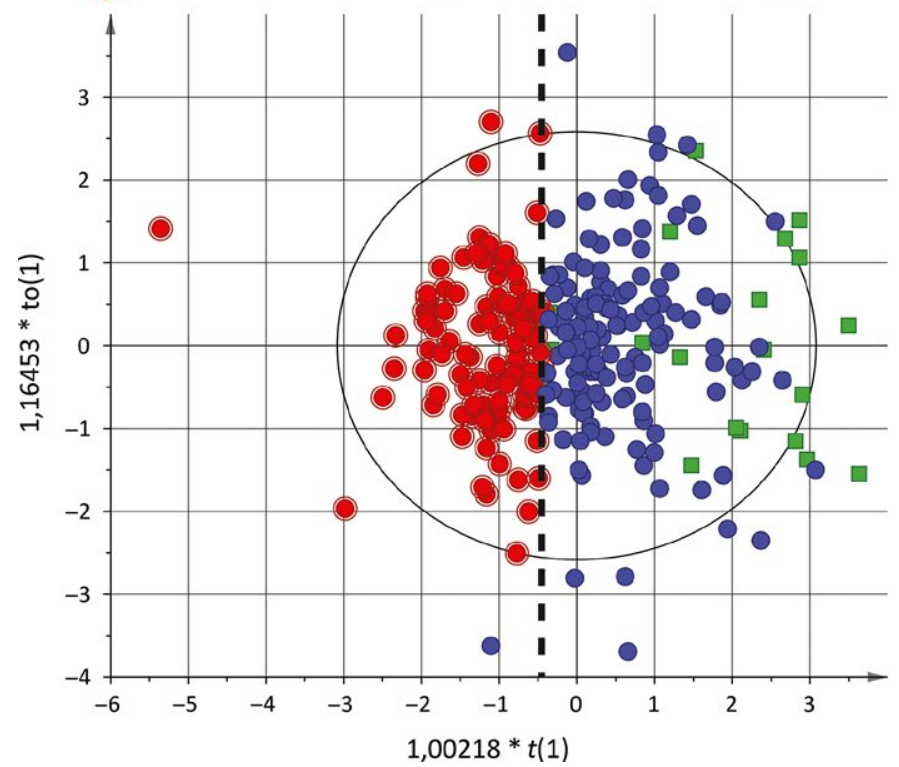

FIGURE 1 Systemic cytokine profiles of immuno-active and immuno-normal irritable bowel syndrome (IBS) patients and healthy subjects (HS). (A) Multivariate orthogonal partial least squares-discriminant analysis (OPLS-DA) score scatter plot showing the discrimination between IBS patients ( $n=244$, blue dots) and HS ( $n=20$, green dots) based on their cytokine profiles, comprising serum levels of interleukin (IL)-6, IL-8, tumor necrosis factor (TNF), and IL-10. (B) The loading scatter plot showing the relationship between the IBS patients and HS and the respective cytokines. (C) OPLS-DA scatter plot depicting the discrimination between IBS patients with elevated serum levels of cytokines (immuno-active, $\mathrm{n}=104$, red dots), IBS patients with normal serum levels of cytokines (immuno-normal $\mathrm{n}=160$, blue dots), and $\mathrm{HS}$ ( $\mathrm{n}=20$ green dots) based on all the analyzed cytokines. Dashed line represents the boundary where IBS patients to the left of this line no longer overlap with HS and thus have divergent serum cytokine profiles

in the pathogenesis or pathophysiology of IBS through effects on other mechanisms.

Cytokines of the innate immune response investigated in this study, ie, IL-1 $\beta$, IL-6, IL-8, TNF, and IL-10 are produced by a range of cells which include but are not limited to, macrophages and mast cells. ${ }^{36,37}$ In this study, we identified an increase in some of these cytokines in IBS patients compared to HS which is in line with the prominent pathophysiology low-grade immune activation hypothesis. ${ }^{12-15,22}$ Measuring only the systemic levels of cytokines we could not define their source and while both macrophages and mast cells for example have been demonstrated as being decreased in IBS patients compared to $\mathrm{HS},{ }^{38}$ a greater number of studies present that these cells and other cells which also produce these cytokines, eg, T lymphocytes are increased in IBS patients. ${ }^{12,39,40}$ These conflicting 

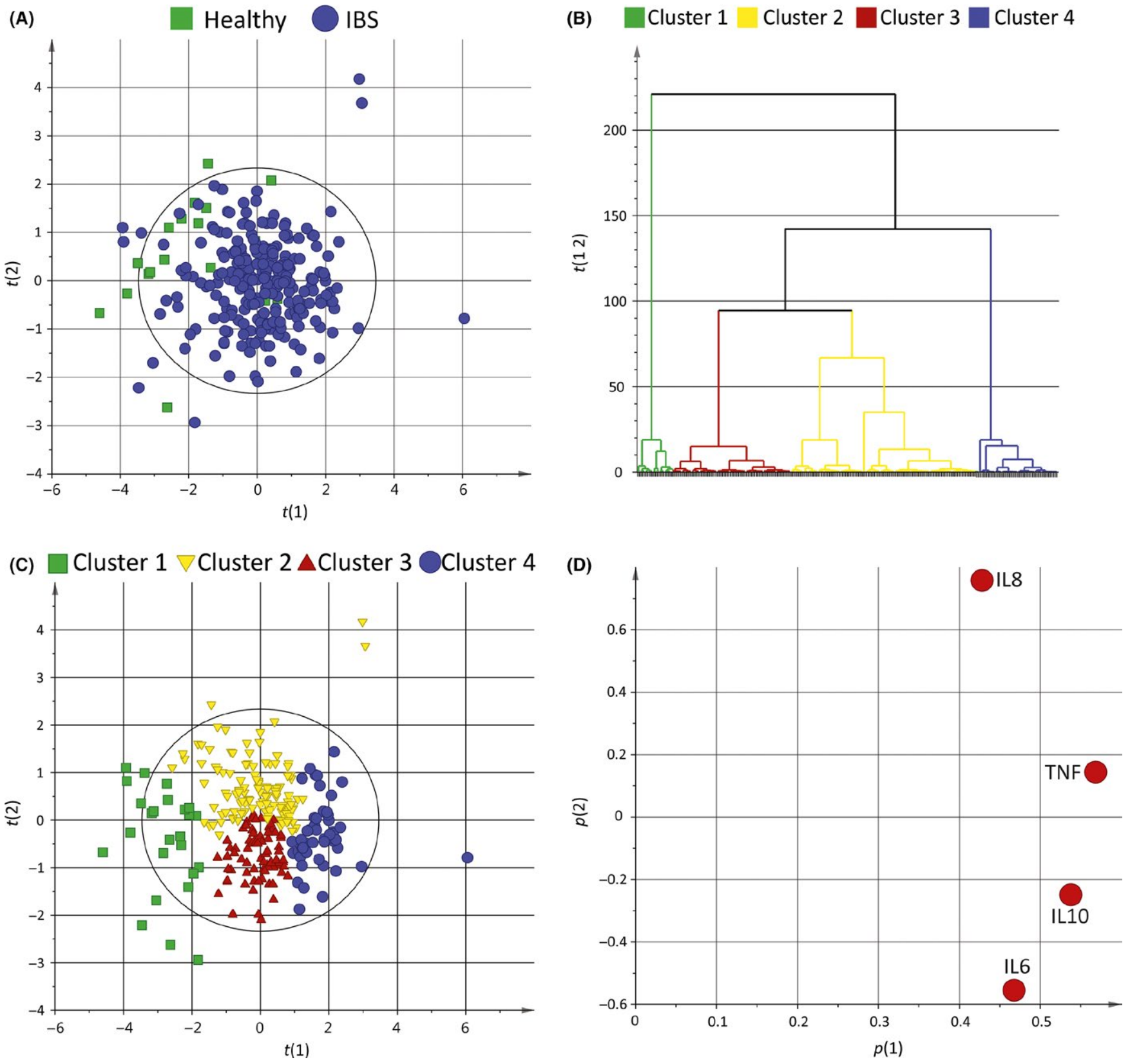

FIGURE 2 Systemic cytokine profiles of irritable bowel syndrome (IBS) patients and healthy subjects (HS). (A) Multivariate principal component analysis (PCA) score scatter plot based on serum levels of interleukin (IL)-6, IL-8, tumor necrosis factor (TNF), and IL-10 levels showing IBS patients ( $n=244$, blue dots) and HS ( $n=20$, green squares). (B) Dendrogram of the unsupervised "bottom-up" hierarchical cluster analysis (HCA) performed based on serum level cytokine profiles. (C) PCA score scatter plot based on serum cytokine levels showing IBS patient and HS clusters as defined by the HCA; cluster 1 as green squares, cluster 2 as yellow inverted triangles, cluster 3 as red triangles, and cluster 4 as blue circles. (D) Loading scatter plot showing the relationship between the respective cytokines

results may be due to heterogeneity among IBS patients and varying techniques and thus future studies measuring systemic or local levels of cytokines may want to consider measurement of immune cells in an attempt to identify the source of potentially increased cytokines. Another potential cause for an increase in systemic cytokines not investigated in this study is the gut microbiota, a factor under much investigation in IBS. ${ }^{41} \mathrm{~A}$ microbiota composition which differs from that of a HS is said to be in dysbiosis and may occur as the result of prior infection, antibiotic use, or diet. ${ }^{42}$ Studies have demonstrated that dysbiosis is present in some patients ${ }^{43}$ and that this may produce the low-grade immune activation identified in some patients. ${ }^{44}$

While evidence for a low-grade immune activation in IBS patients is mounting, it has been suggested that only a subset of patients may be experiencing an increased immune activation. ${ }^{16,45}$ In a previous study by our group, serum levels and mucosal expression of pro- and anti-inflammatory cytokines were determined and used to define patients who had an increased immune activity, named immuno-active IBS patients. ${ }^{16}$ In this study, we were able to 
TAB LE 4 Serum cytokine levels in clusters of IBS patients and healthy subjects (HS) based on cytokine profiles

\begin{tabular}{|c|c|c|c|c|}
\hline Cytokine (pg/mL) & Cluster 1 (7 HS/18 IBS) & Cluster 2 (11 HS/105 IBS) & Cluster 3 (2 HS/72 IBS) & Cluster 4 (49 IBS) \\
\hline IL-1 $\beta$ & $0.41(0.18-0.82)^{(4)}$ & $0.59(0.38-0.92)^{(4)}$ & $0.69(0.5-0.92)^{(4)}$ & $1.04(0.73-1.52)$ \\
\hline IL-6 & $1.16(0.65-1.75)^{(3,4)}$ & $1.8(1.34-2.35)^{(3.4)}$ & $2.79(2.08-4.68)^{(4)}$ & $3.81(3.09-4.68)$ \\
\hline IL-10 & $0.24(0.24-0.49)^{(2,3,4)}$ & $0.75(0.46-1.2)^{(3,4)}$ & $1.04(0.79-1.45)^{(4)}$ & $1.96(1.54-2.75)$ \\
\hline TNF & $5.08(3.83-6.55)^{(2,3,4)}$ & $9.31(7.68-11.5)^{(4)}$ & $8.96(8.03-10.4)^{(4)}$ & $13.3(11.6-15.3)$ \\
\hline
\end{tabular}

Kruskal-Wallis test followed by Dunn's multiple comparisons test. Data shown as median (25\%-75\%).

IL: interleukin; TNF: tumor necrosis factor; (2): Post hoc significant differences, lower than cluster 2; (3): Post hoc significant differences, lower than cluster 3; (4): Post hoc significant differences, lower than cluster 4.

TABLE 5 Clinically measured parameters in immuno-active and immuno-normal IBS patients

\begin{tabular}{|c|c|c|c|}
\hline Clinical & $\begin{array}{l}\text { Immuno-active IBS } \\
(\mathrm{n}=104)\end{array}$ & $\begin{array}{l}\text { Immuno-normal IBS } \\
(\mathrm{n}=140)\end{array}$ & $P$-value \\
\hline IBS-SSS & $267(200-325)$ & $255(175-325)$ & .1 \\
\hline $\begin{array}{l}\text { Colorectal pain threshold } \\
\text { (z score) }\end{array}$ & $-0.01(-0.53$ to 0.52$)$ & $-0.27(-0.79$ to 0.65$)$ & .2 \\
\hline $\begin{array}{l}\text { Colorectal urge threshold } \\
\text { (z score) }\end{array}$ & $-0.09(-0.51$ to 0.59$)$ & $-0.09(-0.78$ to 0.26$)$ & .1 \\
\hline RPSQ & $10.5(7.00-13.0)$ & $9.50(6.00-15.0)$ & .8 \\
\hline CMCQ & $5(2.00-11.0)$ & $5(1.00-10.5)$ & .5 \\
\hline BSI depression & $52.0(41.5-61.0)$ & $48.0(40.0-61.0)$ & .7 \\
\hline BSI anxiety & $50.0(45.0-60.3)$ & $48.0(38.0-59.0)$ & .5 \\
\hline Catastrophizing score & $5.00(2.00-11.0)$ & $5.00(1.00-10.5)$ & .5 \\
\hline Baseline motility index & $280(158-655)$ & $343(201-580)$ & .5 \\
\hline Distension motility index & 949 (432-1394) & $849(623-1256)$ & .9 \\
\hline Recovery motility index & $480(243-795)$ & $508(282-704)$ & 6 \\
\hline Post-meal motility index & $468(295-866)$ & $512(328-877)$ & .7 \\
\hline IBS subtype (\%) (C/D/M) & $17 / 23 / 64$ & $17 / 27 / 96$ & .2 \\
\hline
\end{tabular}

Data shown as median (25\%-75\%).

IBS-SSS: irritable bowel syndrome severity scoring system; BSI: brief symptom inventory; RPSQ: recent physical symptoms questionnaire; C: IBS with constipation; CMCQ: comorbid medical conditions questionnaire; D: IBS with diarrhea; M: IBS mixed constipation and diarrhea.

a Mann-Whitney $U$ test. demonstrate in a second cohort the existence of the previously proposed "immuno-active" subset of patients through OPLS-DA based on serum level of cytokines. ${ }^{16}$ However, in the current study, we recognized that an OPLS-DA requires predefining of the groups in this case immuno-normal and immuno-active. This means that unlike in a PCA whereby no presumptions are made, the OPLS-DA model is influenced by the user and is thus not an unmodified view of the data. Additionally, the identification of the immuno-active subset of patients by this method is only possible by the inclusion of $\mathrm{HS}$ and cannot be used to discriminate patients with an increased immune activity from those with a "normal" level of immune activity if they have not already been predefined. We thus investigated if patients with an increased immune activity could be identified using an unsupervised method indicating the presence of natural underlying differences, or if their prior identification was in part due to the supervised method. The unsupervised method of HCA and PCA, also identified an immuno-active cluster; however, this cluster comprised only $20 \%$ of all patients compared to the $40 \%$ identified though the supervised method. It could be argued that the supervised method was too tolerant in defining patients as immuno-active or that the unsupervised method too restrictive. However, both methods identified a distinct cluster of patients with higher cytokine levels compared to the other IBS patients and HS. Thus, our findings strongly support the existence of an immuno-active subset of IBS patients with future studies now needed to determine cytokine cutoffs to identify patients who are immune-activated.

The lack of increased systemic cytokines in immuno-normal patients suggests another underlying biological or psychosocial mechanism for their symptoms. However, we found neither strong associations between immune activation and IBS symptoms in the immuno-active group nor any other clinical characteristic that characterized them. While immuno-active IBS patients had similar 
TAB L E 6 Comparing clinical parameters between clusters of IBS patients based on serum cytokine profiles

\begin{tabular}{|c|c|c|c|c|c|}
\hline Clinical & Cluster $1(n=18)$ & Cluster $2(n=105)$ & Cluster $3(n=72)$ & Cluster $4(n=49)$ & $P$-value ${ }^{a}$ \\
\hline $\begin{array}{l}\text { Colorectal pain } \\
\text { threshold (z score) }\end{array}$ & $-1.03(-1.58 \text { to }-0.27)^{(2,3,4)}$ & $-0.27(-0.79$ to 0.78$)$ & $-0.01(-0.63$ to 0.52$)$ & $-0.01(-0.53$ to 0.47$)$ & .01 \\
\hline $\begin{array}{l}\text { Colorectal urge } \\
\text { threshold (z score) }\end{array}$ & $-0.44(-1.21$ to -0.25$)$ & $-0.09(-0.78$ to 0.94$)$ & $-0.09(-0.78$ to 0.26$)$ & $-0.09(-0.44$ to 0.59$)$ & .4 \\
\hline $\begin{array}{l}\text { Comorbid medical } \\
\text { conditions } \\
\text { questionnaire }\end{array}$ & $1(0.5-3)$ & $2(1-3)$ & $1(0-3)$ & $2(1-4)$ & .7 \\
\hline BSI depression & $48(40-58)$ & $48(40-61)$ & $58(42-61.3)$ & $56(45-59)$ & .1 \\
\hline $\begin{array}{l}\text { Distension motility } \\
\text { index }\end{array}$ & $756(657-1344)$ & 847 (607-1219) & 874 (474-1378) & 981 (489-1367) & .9 \\
\hline $\begin{array}{l}\text { Recovery motility } \\
\text { index }\end{array}$ & $420(275-568)$ & $493(282-711)$ & 445 (247-771) & 479 (253-794) & .9 \\
\hline $\begin{array}{l}\text { Post-meal motility } \\
\text { index }\end{array}$ & $471(290-861)$ & 497 (311-913) & 470 (319-829) & $423(201-817)$ & .7 \\
\hline $\begin{array}{l}\text { IBS subtype (\%) } \\
\text { (C/D/M) }\end{array}$ & $11 / 22 / 67$ & $19 / 17 / 67$ & $6 / 21 / 74$ & $19 / 30 / 51$ & .07 \\
\hline
\end{tabular}

Data shown as median (25\%-75\%).

IBS-SSS: irritable bowel syndrome severity scoring system; BSI: brief symptom inventory; C: IBS with constipation; D: IBS with diarrhea; M: IBS mixed constipation and diarrhea; (2): Post hoc significant differences, lower than cluster 2; (3): Post hoc significant differences, lower than cluster 3; (4): Post hoc significant differences, lower than cluster 4.

${ }^{a}$ Kruskal-Wallis test followed by Dunn's multiple comparisons test.

symptom pattern and severity compared to immuno-normal patients questioning the relevance of immune activation toward the pathophysiology of IBS it does not remove potential influence of the increased immune activation in the pathogenesis of IBS in these patients. Even though our data seem to suggest there are no direct effects of immune factors on symptoms, indirect effects via other factors cannot be excluded. Further investigation should hence focus on what causes immune activation in this subset of patients and how this might influence IBS symptomatology.

It has been reported that as many as $45 \%$ of IBS patients have other comorbid conditions. ${ }^{11}$ Although the nature of the comorbidity was not distinguished with accuracy, a previous study demonstrated that patients with comorbidities have increased systemic proinflammatory IL-6, IL-8, and characteristically IL-1 $\beta$ and TNF. ${ }^{46}$ Thus, it is possible that the cause behind the increased systemic immune activation is not linked to the IBS symptoms but to comorbidities per se. The number of comorbid medical conditions, somatization (the tendency to report multiple somatic symptoms), and anxiety and depression (common comorbid conditions with IBS and associated with increased IL- $6^{24}$ ) were considered in this study, but few if any associations were found. Thus, while this study did not find any strong association between cytokine levels and comorbidities in IBS, their inclusion should be taken in detail during patient assessment and considered during data analysis due to the impact comorbid diseases or disorders can have on the host.

Although previous studies have demonstrated correlations of IL- 6 to symptoms of IBS, ${ }^{22,24}$ this and our previous study identified only tendencies in that direction within the 2 large IBS cohorts. ${ }^{16}$ Furthermore, the correlations of anti-inflammatory IL-10 with quality of life and IBS-SSS may be coincidental and not causational, as the secretion of IL-10 increases in an immunoregulatory manner as levels of pro-inflammatory factors rise. Thus, the lack of correlations between systemic cytokine level and symptoms in this and our previous study suggests that an increased immune activation is not directly related to symptom characteristics. ${ }^{16}$

Theoretically, patients with an increased immune activity should benefit from targeted treatment. However, based on our current results, reducing inflammation is not expected to be helpful in reducing IBS symptoms. Anti-inflammatory drugs decreasing the synthesis of prostaglandins (modulators of the inflammatory response ${ }^{47}$ ), eg, mesalazine, have had partial success in relief of symptoms including abdominal pain in small subsets of patients, but the primary endpoints 
in clinical trials were negative. ${ }^{45,48,49}$ A defining characteristic of immuno-active IBS is higher systemic IL-6. Anti-inflammatory drugs that decrease the synthesis of prostaglandins, ie, non-steroidal antiinflammatory drugs, may be considered as a treatment options for IBS. However, enthusiasm is hampered by their $\mathrm{GI}$ side effect profile ${ }^{50}$ and they may even exacerbate or cause IBS. ${ }^{51}$ Therefore, the usefulness of anti-inflammatory treatments in IBS remains uncertain. It may be the case that both IBS symptoms and increased inflammation are driven by a third factor, and addressing this factor would reduce both. Further studies are needed to elucidate the role of immune activation in IBS, before considering anti-inflammatory treatments.

Although extensive, this study has several limitations. As the samples were collected and assed for cytokine levels over the last decade, we used the ROME II criteria to define IBS. Therefore, our results may have been different in a cohort based on the recently launched Rome IV criteria for IBS, ${ }^{2}$ but most IBS patients who seek health care for their symptoms in Western countries who fulfill older diagnostic criteria for IBS also fulfill the Rome IV criteria for IBS. ${ }^{52,53}$ Based on this we do not think that the use of different diagnostic criteria for IBS would have had any major influence on our results. Considering the many IBS patients included, the number of HS is comparatively small; however, the high homogeneity of the group indicates that additional HS would likely have little impact on the findings of this study. More women than men have autoimmune diseases, ${ }^{54}$ and although conflicting, findings from studies investigating systemic levels of cytokines between males and females ${ }^{55,56}$ should be considered with regard to the differing gender distribution in this study between patients and HS. However, unlike with the high immune activation of autoimmune diseases, studies are lacking which investigate gender differences in prominence of low grade immune activation as indicated in IBS. Additionally, our findings are in line with our previously gender balanced study. ${ }^{16}$ Furthermore, as the study is cross-sectional, it cannot determine if cytokines have delayed effects on symptoms. Finally, serum cytokines may be increased for other reasons than IBS. However, in our previous study we also examined mRNA expressions of cytokines in bowel biopsies and found similar results. It may be that other inflammatory indicators could be of more relevance than cytokines and this requires future study.

In summary, the increased levels of systemic serum cytokines recorded in patients compared to HS could not be used to discriminate patients from HS. However, supervised and unsupervised statistical modeling both identified a subset of IBS patients (20\%-40\%) with increased systemic immune activity, which is in line with our previous study. ${ }^{16}$ However, these patients were indistinguishable from patients with normal immune activity based on symptom severity, psychological factors, or colorectal sensorimotor function, indicating no direct relationship between a more active immune system and symptoms in IBS. Thus, future studies should focus on elucidating the mechanisms behind the increased immune activity in a subset of patients, and the relevance in the pathophysiology or even pathogenesis of IBS through interaction with factors not investigated in this study.

\section{DISCLOSURE}

Magnus Simrén has served as a consultant/ Advisory Board member for AstraZeneca, Danone Nutricia, Nestle, Almirall, Allergan, Albireo, Menarini, Glycom and Shire, and as a speaker for Tillotts, Takeda, Shire, Menarini, Allergan and Almirall, and has received unrestricted research grants from Danone Nutricia and Ferring Pharmaceuticals. Dr van Tilburg has received investigator initiated research support from Takeda.

\section{AUTHOR CONTRIBUTION}

SB was responsible for study design, analysis, and interpretation of data, drafting of the manuscript, statistical analysis; OP, WEW, and MS was responsible for study concept and design, acquisition of data, critical revision of the manuscript for important intellectual content, obtained funding; DAB and HT contributed to acquisition of data, critical revision of the manuscript for important intellectual content; LÖ contributed to critical revision of the manuscript for important intellectual content; MS has served as a consultant/Advisory Board member for AstraZeneca, Danone Nutricia, Nestle, Almirall, Allergan, Albireo, Menarini, Glycom and Shire, and as a speaker for Tillotts, Takeda, Shire, Menarini, Allergan and Almirall, and has received unrestricted research grants from Danone Nutricia and Ferring Pharmaceuticals; MALvT was responsible for study concept and design, analysis and interpretation of data, drafting of the manuscript, critical revision of the manuscript for important intellectual content, obtained funding, study supervision. Dr van Tilburg has received investigator initiated research support from Takeda.

\section{ORCID}

\section{S. M. P. Bennet (iD http://orcid.org/0000-0003-4549-5150 \\ W. E. Whitehead iD http://orcid.org/0000-0001-6587-7602}

\section{REFERENCES}

1. Drossman DA, Camilleri M, Mayer EA, Whitehead WE. AGA technical review on irritable bowel syndrome. Gastroenterology. 2002;123:2108-2131.

2. Lacy BE, Mearin F, Chang L, et al. Bowel disorders. Gastroenterology. 2016;150:1393-407.e5.

3. Palsson OS, Whitehead WE, van Tilburg MAL, et al. Development and validation of the Rome IV diagnostic questionnaire for adults. Gastroenterology. 2016;150:1481-1491.

4. Lovell RM, Ford AC. Global prevalence of and risk factors for irritable bowel syndrome: a meta-analysis. Clin Gastroenterol Hepatol. 2012;10:712-21.e4.

5. Sperber AD, Dumitrascu D, Fukudo $S$, et al. The global prevalence of IBS in adults remains elusive due to the heterogeneity of studies: a Rome Foundation working team literature review. Gut. 2017; 66:1075-1082.

6. Plavšić I, Hauser G, Tkalčić M, Pletikosić S, Salkić N. Diagnosis of irritable bowel syndrome: role of potential biomarkers. Gastroenterol Res Pract. 2015;2015:490183. 
7. Boeckxstaens GE, Drug V, Dumitrascu D, et al. Phenotyping of subjects for large scale studies on patients with IBS. Neurogastroenterol Motil. 2016;28:1134-1147.

8. Palsson OS, Baggish JS, Turner MJ, Whitehead WE. IBS patients show frequent fluctuations between loose/watery and hard/ lumpy stools: implications for treatment. Am J Gastroenterol. 2012;107:286-295. https://doi.org/10.1038/ajg.2011.358.

9. Bajor A, Tornblom H, Rudling M, Ung KA, Simren M. Increased colonic bile acid exposure: a relevant factor for symptoms and treatment in IBS. Gut. 2015;64:84-92.

10. Marciani L, Cox EF, Hoad CL, et al. Postprandial changes in small bowel water content in healthy subjects and patients with irritable bowel syndrome. Gastroenterology. 2010;138:469-477, 77 e1.

11. Whitehead WE, Palsson O, Jones KR. Systematic review of the comorbidity of irritable bowel syndrome with other disorders: what are the causes and implications? Gastroenterology. 2002;122:1140-1156.

12. Ohman L, Isaksson S, Lindmark AC, et al. T-cell activation in patients with irritable bowel syndrome. Am J Gastroenterol. 2009;104:1205-1212.

13. Cremon C, Gargano L, Morselli-Labate AM, et al. Mucosal immune activation in irritable bowel syndrome: gender-dependence and association with digestive symptoms. Am J Gastroenterol. 2009;104:392-400.

14. Liebregts $T$, Adam B, Bredack C, et al. Immune activation in patients with irritable bowel syndrome. Gastroenterology. 2007;132:913-920.

15. Seyedmirzaee S, Hayatbakhsh MM, Ahmadi B, et al. Serum immune biomarkers in irritable bowel syndrome. Clin Res Hepatol Gastroenterol. 2016;40:631-637.

16. Bennet SM, Polster A, Tornblom H, et al. Global cytokine profiles and association with clinical characteristics in patients with irritable bowel syndrome. Am J Gastroenterol. 2016;111:1165-1176.

17. Vázquez-Frias R, Gutiérrez-Reyes $G$, Urbán-Reyes $M$, et al. Proinflammatory and anti-inflammatory cytokine profile in pediatric patients with irritable bowel syndrome. Rev Gastroenterol Méx (English Edition). 2015;80:6-12.

18. Chang L, Adeyemo M, Karagiannides I, et al. Serum and colonic mucosal immune markers in irritable bowel syndrome. Am J Gastroenterol. 2012;107:262-272.

19. Scheller J, Chalaris A, Schmidt-Arras D, Rose-John S. The pro- and anti-inflammatory properties of the cytokine interleukin-6. Biochim Biophys Acta. 2011;1813:878-888.

20. Shahzad A, Knapp M, Lang I, Köhler G. Interleukin 8 (IL-8) - a universal biomarker? Int Arch Med. 2010;3:11.

21. Parameswaran N, Patial S. Tumor necrosis factor-alpha signaling in macrophages. Crit Rev Eukaryot Gene Expr. 2010;20:87-103.

22. Clarke G, Quigley EM, Cryan JF, Dinan TG. Irritable bowel syndrome: towards biomarker identification. Trends $\mathrm{Mol}$ Med. 2009;15:478-489.

23. Hughes PA, Harrington AM, Castro J, et al. Sensory neuro-immune interactions differ between irritable bowel syndrome subtypes. Gut. 2013;62:1456-1465.

24. Dinan TG, Clarke G, Quigley EM, et al. Enhanced cholinergicmediated increase in the pro-inflammatory cytokine IL- 6 in irritable bowel syndrome: role of muscarinic receptors. Am J Gastroenterol. 2008;103:2570-2576.

25. Rana SV, Sharma S, Sinha SK, Parsad KK, Malik A, Singh K. Proinflammatory and anti-inflammatory cytokine response in diarrhoea-predominant irritable bowel syndrome patients. Trop Gastroenterol. 2012;33:251-256.

26. Drossman D. The functional gastrointestinal disorders and the Rome II process. Gut. 1999;45(Suppl 2):II1-II5.

27. Drossman DA. The functional gastrointestinal disorders and the Rome III process. Gastroenterology. 2006;130:1377-1390.
28. Kanazawa M, Palsson OS, Thiwan SIM, et al. Contributions of pain sensitivity and colonic motility to IBS symptom severity and predominant bowel habits. Am J Gastroenterol. 2008;103:2550-2561.

29. Francis CY, Morris J, Whorwell PJ. The irritable bowel severity scoring system: a simple method of monitoring irritable bowel syndrome and its progress. Aliment Pharmacol Ther. 1997;11:395-402.

30. Patrick DL, Drossman DA, Frederick IO, DiCesare J, Puder KL. Quality of life in persons with irritable bowel syndrome: development and validation of a new measure. Dig Dis Sci. 1998;43:400-411.

31. Drossman DA, Patrick DL, Whitehead WE, et al. Further validation of the IBS-QOL: a disease-specific quality-of-life questionnaire. Am J Gastroenterol. 2000;95:999-1007.

32. MacLean EW, Palsson OS, Turner MJ, Whitehead WE. Development and validation of new disease-specific measures of somatization and comorbidity in IBS. J Psychosom Res. 2012;73:351-355. https:// doi.org/10.1016/j.jpsychores.2012.08.007.

33. Derogatis LR. BSI 18, Brief Symptom Inventory 18: Administration, Scoring and Procedures Manual. Minneapolis, MN: NCS Pearson, Inc.; 2001.

34. Keefe FJ, Brown GK, Wallston KA, Caldwell DS. Coping with rheumatoid arthritis pain: catastrophizing as a maladaptive strategy. Pain. 1989;37:51-56.

35. Umetrics. SIMCA-P and multivariate analysis FAQ, Version 1.01. http://umetrics.com/sites/default/files/kb/multivariate_faq.pdf. Accessed September 26, 2016.

36. Zhang L, Song J, Hou X. Mast cells and irritable bowel syndrome: from the bench to the bedside. J Neurogastroenterol Motil. 2016;22:181-192.

37. Dinarello CA. Historical review of cytokines. Eur J Immunol. 2007;37(Suppl 1):S34-S45.

38. Braak B, Klooker TK, Wouters MM, et al. Mucosal immune cell numbers and visceral sensitivity in patients with irritable bowel syndrome: is there any relationship? Am J Gastroenterol. 2012;107:715-726.

39. O'Sullivan M, Clayton N, Breslin NP, et al. Increased mast cells in the irritable bowel syndrome. Neurogastroenterol Motil. 2000;12:449-457.

40. Chadwick VS, Chen W, Shu D, et al. Activation of the mucosal immune system in irritable bowel syndrome. Gastroenterology. 2002;122:1778-1783.

41. Bennet SM, Ohman L, Simren M. Gut microbiota as potential orchestrators of irritable bowel syndrome. Gut Liv. 2015;9:318-331.

42. Bennet SMP, Bohn L, Storsrud S, et al. Multivariate modelling of faecal bacterial profiles of patients with IBS predicts responsiveness to a diet low in FODMAPs. Gut. 2018;67:872-881.

43. Casén C, Vebø HC, Sekelja M, et al. Deviations in human gut microbiota: a novel diagnostic test for determining dysbiosis in patients with IBS or IBD. Aliment Pharmacol Ther. 2015;42:71-83.

44. Collins SM, Denou E, Verdu EF, Bercik P. The putative role of the intestinal microbiota in the irritable bowel syndrome. Dig Liver Dis. 2009;41:850-853.

45. Barbara G, Cremon C, Annese V, et al. Randomised controlled trial of mesalazine in IBS. Gut. 2016;65:82-90.

46. Scully P, McKernan DP, Keohane J, et al. Plasma cytokine profiles in females with irritable bowel syndrome and extra-intestinal comorbidity. Am J Gastroenterol. 2010;105:2235-2243.

47. Ricciotti E, FitzGerald GA. Prostaglandins and Inflammation. Arterioscler Thromb Vasc Biol. 2011;31:986-1000.

48. Lam C, Tan W, Leighton M, et al. A mechanistic multicentre, parallel group, randomised placebo-controlled trial of mesalazine for the treatment of IBS with diarrhoea (IBS-D). Gut. 2016;65:91-99. 
49. Dunlop SP, Jenkins D, Neal KR, et al. Randomized, double-blind, placebo-controlled trial of prednisolone in post-infectious irritable bowel syndrome. Aliment Pharmacol Ther. 2003;18:77-84.

50. Bjarnason I, Hayllar J, MacPherson AJ, Russell AS. Side effects of nonsteroidal anti-inflammatory drugs on the small and large intestine in humans. Gastroenterology. 1993;104:1832-1847.

51. Keszthelyi D, Dackus GH, Masclee GM, Kruimel JW, Masclee AA. Increased proton pump inhibitor and NSAID exposure in irritable bowel syndrome: results from a case-control study. BMC Gastroenterol. 2012;12:121.

52. Vork L, Weerts Z, Mujagic Z, et al. Rome III vs Rome IV criteria for irritable bowel syndrome: a comparison of clinical characteristics in a large cohort study. Neurogastroenterol Motil. 2018;30:e13189. https://doi.org/10.1111/nmo.13189.

53. Aziz I, Tornblom H, Palsson OS, Whitehead WE, Simren M. How the change in IBS criteria from Rome III to Rome IV impacts on clinical characteristics and key pathophysiological factors. Am J Gastroenterol Suppl. 2018 (accepted manuscript).

54. Fairweather D, Frisancho-Kiss S, Rose NR. Sex differences in autoimmune disease from a pathological perspective. Am J Pathol. 2008;173:600-609.
55. Crockett ET, Spielman W, Dowlatshahi S, He J. Sex differences in inflammatory cytokine production in hepatic ischemia-reperfusion. J Inflamm. 2006;3:16.

56. Bouman A, Heineman MJ, Faas MM. Sex hormones and the immune response in humans. Hum Reprod Update. 2005;11:411-423.

\section{SUPPORTING INFORMATION}

Additional supporting information may be found online in the Supporting Information section at the end of the article. 